This document is the accepted manuscript version of the following article: Balena, A., Perul1i, A., Fernandez, M., De Giorgi, M. L., Nedelcu, G., Kovalenko, M. V., \& Anni, M. (2018). Temperature dependence of the amplified spontaneous emission from CsPbBr3 nanocrystal thin films. Journal of Physical Chemistry C, 122(10), 5813-5819. https://doi.org/10.1021/acs.jpcc.8b01419

\title{
Temperature Dependence of the Amplified
}

\section{Spontaneous Emission from $\mathrm{CsPbBr}_{3}$ Nanocrystal}

\section{Thin Films}

\author{
Antonio Balena, ${ }^{\dagger}$ Andrea Perulli, ${ }^{\dagger}$ Manuel Fernandez, ${ }^{\dagger}$ Maria Luisa De Giorgi, ${ }^{\dagger}$ \\ Georgian Nedelcu, Maksym V. Kovalenko, ${ }^{\ddagger}, 9$ and Marco Anni ${ }^{*, \dagger}$ \\ †Dipartimento di Matematica e Fisica "Ennio De Giorgi",Università del Salento, Via per \\ Arnesano, 73100 Lecce, Italy \\ $\ddagger$ Institute of Inorganic Chemistry, Department of Chemistry and Applied Bioscience, ETH \\ Zürich, CH-8093 Zürich, Switzerland \\ \Laboratory for Thin Films and Photovoltaics, Empa - Swiss Federal Laboratories for \\ Materials Science and Technology, CH-8600 Dübendorf, Switzerland \\ E-mail: marco.anni@unisalento.it \\ Phone: +39 0832297540
}




\begin{abstract}
Cesius lead halide perovskite colloidal nanocrystals are among the most promising perovskite systems for light emitting devices applications, due to their high fluorescence quantum yield and high optical gain at room temperature. In this Letter, we report on the first investigation of the temperature dependence of the Amplified Spontaneous Emission (ASE) properties of thin films of $\mathrm{CsPbr}_{3}$ nanocrystals. We demonstrate that ASE is strongly temperature dependent, with a complex variation in temperature of the ASE intensity, threshold, and peak wavelength. The joint investigation of the photoluminescence (PL) spectra below and above the ASE threshold allows us to conclude that the temperature increase results in the formation of disordered sub-domains emitting in the low energy tail of the PL spectra, leading to the existence of three emission regimes with transitions at about $90 \mathrm{~K}$ and about $170 \mathrm{~K}$, with individually different temperature dependences.
\end{abstract}

\title{
Introduction
}

Lead halide perovskite thin films are receiving an increasing attention in the last few years, initially stimulated by the impressive performances improvement of solar cells, recently reaching a record power conversion efficiency of $22.7 \% .{ }^{1}$ Beyond the excellent photovoltaic properties, allowed by the combination of high absorption cross sections, large carrier diffusion length and low trap density, ${ }^{2,3}$ lead halide perovskites also show excellent optical properties, like high photoluminescence Quantum Yield (QY), ${ }^{4}$ fully tunable emission from the blue to the near infrared, ${ }^{4-6}$ by adjusting the material chemical composition ${ }^{7,8}$ and exploiting the quantum confinement in nanostructures, low Amplified Spontaneous Emission (ASE) threshold and high optical gain at room temperature. ${ }^{9,10}$ These properties, together with the possibility of deposition from solution, have been recently exploited for the realization of the first optically pumped perovskite lasers, using different cavity geometries like vertical microcavity lasers,${ }^{11}$ whispering gallery mode lasers, ${ }^{10}$ random lasers ${ }^{12}$ and Distributed 
Feedback lasers. ${ }^{13}$

The high applied interest of the solution-processed perovskites thin films also stimulated the research on the basic photophysical processes affecting the material properties. In this frame the investigation of the optical properties as a function of the temperature, starting from cryogenic temperatures, is a very powerful tool, and it has been to date exploited to investigate the effects of the variation of the excitation-phonon coupling, ${ }^{14,15}$ of trapping or detrapping processes, ${ }^{14,16}$ of exciton dissociation ${ }^{15,17}$ and of crystalline phase variation on the absorption, ${ }^{18,19}$ the photoluminescence, ${ }^{14-17,20-23}$ and the excitation relaxation dynamics ${ }^{14,16,17,21-23}$ of perovskite thin films.

Despite the rich scenario of temperature-induced processes affecting the basic photophysics of perovskite thin films, the ASE temperature dependence investigation is to date limited to few experiments, on polycrystalline bulk thin films of $\mathrm{MAPbI}_{3}{ }^{24,25}$ and of $\mathrm{CsPbBr}_{3},{ }^{26}$ evidencing effects of the crystalline phase transitions and of the exciton trapping and detrapping processes, respectively. On the contrary the temperature dependence of the ASE of thin films of colloidal perovskite nanocrystals has been to date not investigated yet.

In this paper, we report on the first investigation of the temperature dependence of the Amplified Spontaneous Emission properties of a thin film of $\mathrm{CsPbBr}_{3}$ nanocrystals between $10 \mathrm{~K}$ and $290 \mathrm{~K}$, excited by a Nitrogen laser (3 ns pulses at $337 \mathrm{~nm}$, repetition rate $10 \mathrm{~Hz}$ ). We demonstrate that the temperature decrease to $10 \mathrm{~K}$ results in a considerable increase of the optical gain, and in a strong decrease of the ASE threshold. A complex temperature dependence of the ASE peak energy, intensity and threshold is observed, evidencing the presence of three different emissions regimes, namely below $90 \mathrm{~K}$, between $90 \mathrm{~K}$ and $170 \mathrm{~K}$, and above $170 \mathrm{~K}$, with individually different temperature dependence. The joint investigation of the emission properties above and below the ASE threshold indicates the formation of new emitting species at low energy at characteristic temperatures of $90 \mathrm{~K}$ and $170 \mathrm{~K}$, ascribed to partially disordered crystalline sub-domains. We demonstrate that the ASE properties of the films are strongly affected by the formation of these domains, and are mainly determined 
by the emission properties of the species emitting at lower energy.

\section{Experimental section}

\section{Nanocrystal synthesis, thin film deposition and characterization}

The $\mathrm{CsPbBr}_{3}$ nanocrystals have been synthesized by following the report of Protesescu et al. ${ }^{4}$ but with some modifications. The detailed procedure is described in the Supporting Information.

The photoluminescence Quantum Yield (PLQY) has been measured in solution against Fluorescein in $\mathrm{NaOH} 0.1 \mathrm{M}$ (excitation wavelength $488 \mathrm{~nm}$ ). ${ }^{27}$

The nanocrystals shape and dimensions have been investigated by using a Hitachi HT7700 EXALENS microscope operated at $100 \mathrm{kV}$.

The active film has been deposited by drop casting on glass substrates, at room temperature and in air. In order to improve the film morphology the film was left to dry under a Petri dish, thus slowing the solvent evaporation.

The film morphology has been investigated by a JEOL JSM-6480LV SEM measurements, operated at $10 \mathrm{kV}$.

The fluorescence maps were measured by a Nikon Eclipse C1 fluorescence inverted microscope (20X DIC Plan Apochromat objective, 0.50 numerical aperture), by exciting with a spectrally filtered mercury lamp in the range 470-490 $\mathrm{nm}$ and collecting the fluorescence in the range $520-560 \mathrm{~nm}$.

\section{PL, Amplified Spontaneous Emission and gain measurements}

A LTB MNL 100 nitrogen laser, delivering 3 ns pulses at $355 \mathrm{~nm}$, with a peak energy up to $155 \mu \mathrm{J}$ was used as excitation source of the PL, ASE and optical gain measurements. The pump laser has been focused by a cylindrical lens on a rectangular stripe with length up to $4 \mathrm{~mm}$ and width of $100 \mu \mathrm{m}$. The laser excitation density has been varied by a variable neutral 


\section{Results and discussion}

As a first step in our experiment, we investigated the excitation density dependence of the PL spectra, at room temperature $(290 \mathrm{~K})$. The PL spectrum shows, at low excitation density, a single emission resonance, peaked at $513.7 \mathrm{~nm}(2.414 \mathrm{eV})$ with a full width at half maximum (FWHM) of $22.8 \mathrm{~nm}$ (108 meV, see Fig. 1 a). As the excitation density increases a progressive intensity increase is observed, with a clear lineshape variation due to the appearance of a narrow band peaked at about $526.7 \mathrm{~nm}$, progressively dominating the emission as the excitation density is further increased. These features are comparable to the ones previously reported in similar samples both under femtosecond and nanosecond pumping, ${ }^{10}$ and are ascribed to ASE. The ASE band appearance results in a clear slope variation of the total intensity dependence on the excitation density (see inset of Fig.1 a). We estimated an ASE threshold of about $2.55 \mathrm{mJcm}^{-2}$ by continuously increasing the pump excitation density and determining the minimum excitation density that allows to observe the ASE band appearance in the spectra. The optical gain spectrum, measured with the variable stripe method at an excitation density of $6.0 \mathrm{mJcm}^{-2}$, shows a single peak at 525.6 $\mathrm{nm}$ with a maximum value of $12.9 \mathrm{~cm}^{-1}$ (see Fig. $1 \mathrm{c}$ ).

When the temperature is decreased to $10 \mathrm{~K}$ (see Fig. $1 \mathrm{~b}$ ) we observe, at low excitation density, a PL peak red-shift to $521.5 \mathrm{~nm}(2.377 \mathrm{eV})$ and a FWHM reduction to $7.8 \mathrm{~nm}$ $(35.6 \mathrm{meV})$. As the excitation density increases a clear ASE band is observed, peaked at $530.5 \mathrm{~nm}$, with a strongly reduced threshold of $147 \mu \mathrm{Jcm}^{-2}$. The temperature reduction 
a)

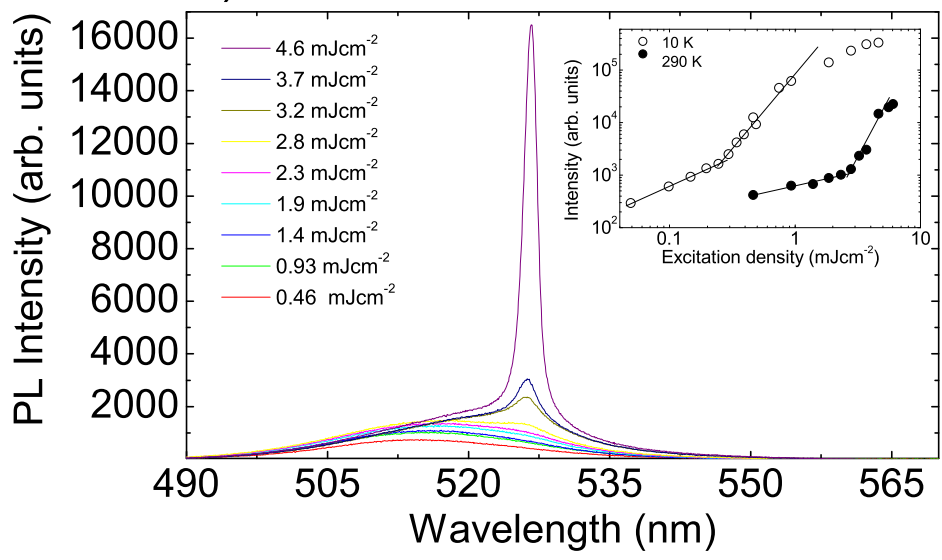

b)

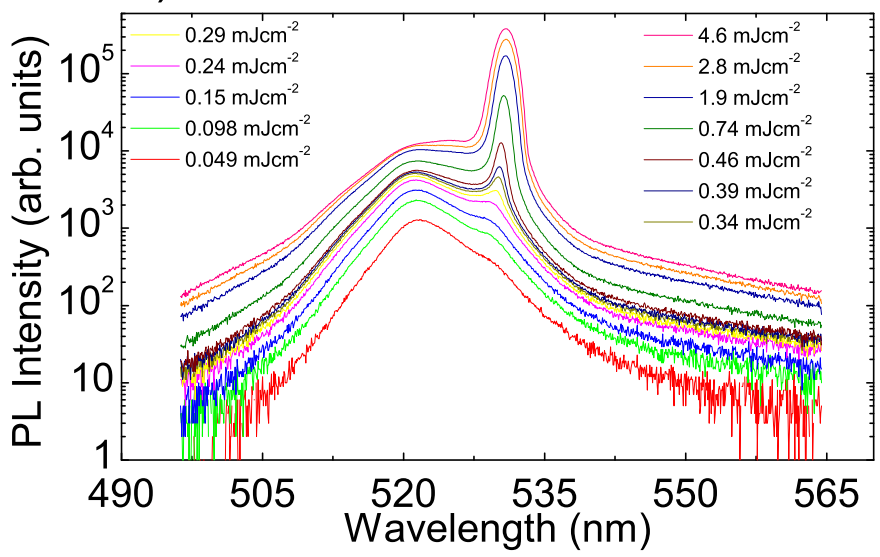

c)

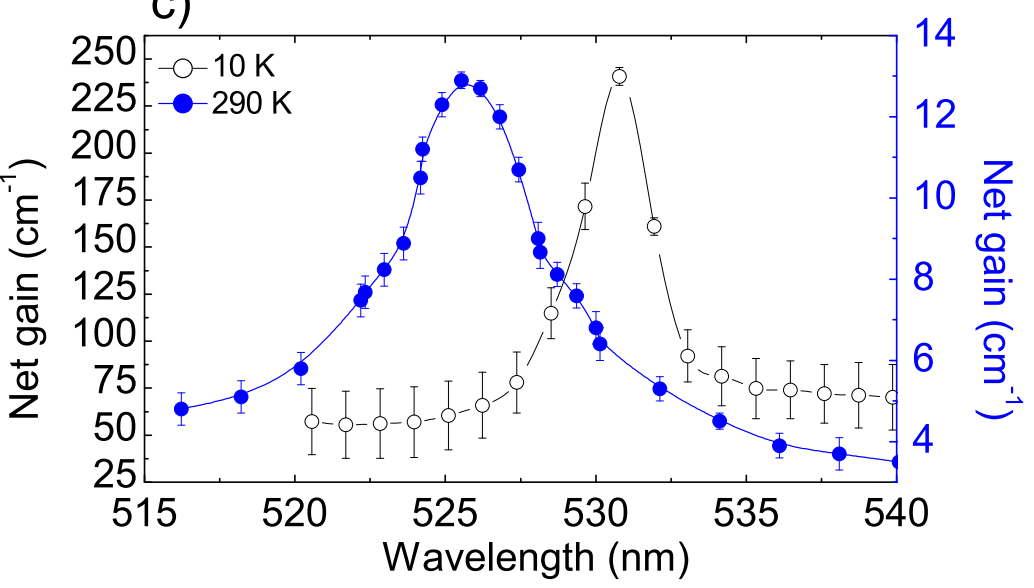

Figure 1: a): Excitation density dependence of the PL spectra at room temperature (290 K). A clear ASE band is visible at $526.7 \mathrm{~nm}$. Inset: excitation density dependence of the total PL intensity, showing the slope increase across the ASE threshold. b): Excitation density dependence of the PL spectra at $\mathrm{T}=10 \mathrm{~K}$. c): net gain spectrum at $\mathrm{T}=10 \mathrm{~K}$ and $\mathrm{T}=290 \mathrm{~K}$.

also leads to a considerable enhancement of the net optical gain, reaching the remarkable maximum value of $241 \mathrm{~cm}^{-1}$ (see Fig. 1 c) at an excitation density of $930 \mu \mathrm{Jcm}^{-2}$. Thus the temperature decrease to $10 \mathrm{~K}$ allows to increase the maximum gain value of about 19 times, 
at an excitation density 6.5 times lower, evidencing a considerable enhancement of the gain properties.

In order to have a clear understanding of the temperature dependent processes affecting the optical gain in our sample we thus measured the emission spectra at a common excitation density of $4.6 \mathrm{mJcm}^{-2}$ (above the room temperature ASE threshold).

The obtained results, reported in Fig. 2 a as a 2D map, show a complex temperature dependence, in particular as the temperature increases we observe that:

1) the ASE peak position shows a progressive linear (in energy) blue shift (see Fig.2 b) up to $90 \mathrm{~K}$ with a best fit slope of $250 \pm 4 \mu \mathrm{eV} / \mathrm{K}$, followed by a weaker blueshift up to $170 \mathrm{~K}$ with a slope of $67 \pm 7 \mu \mathrm{eV} / \mathrm{K}$, then a red-shift up to $270 \mathrm{~K}$, and finally a blue-shift up to $290 \mathrm{~K}$;

2) The integrated spectrum intensity is almost constant up to $50 \mathrm{~K}$ (see Fig. 2 c), then a strong intensity reduction is observed up to $90 \mathrm{~K}$, followed by a weaker quenching up to $170 \mathrm{~K}$. A strong intensity recovery is observed up to $230 \mathrm{~K}$, finally followed by a further quenching up to $290 \mathrm{~K}$.

3) The ASE threshold shows a strong initial increase up to $90 \mathrm{~K}$ (see Fig. 2 c), followed by a weaker increase up to $190 \mathrm{~K}$. Then an abrupt decrease is observed between $190 \mathrm{~K}$ and $210 \mathrm{~K}$, followed by a continuous threshold increase up to $290 \mathrm{~K}$.

Overall, these results evidence the existence of two common temperatures at which the temperature dependences change, namely $90 \mathrm{~K}$ and between $170 \mathrm{~K}$ and $190 \mathrm{~K}$, suggesting the presence of three different emission regimes.

In order to rationalize the previous results we also investigated the PL temperature dependence below the ASE threshold, at a common excitation density of $150 \mu \mathrm{Jcm}^{-2}$.

The PL spectrum at T=10 K (see Fig.3 a) shows a main narrow peak at $521.5 \mathrm{~nm}(2.377$ $\mathrm{eV})$, with a FWHM of $8.6 \mathrm{~nm}(39.2 \mathrm{meV})$. 


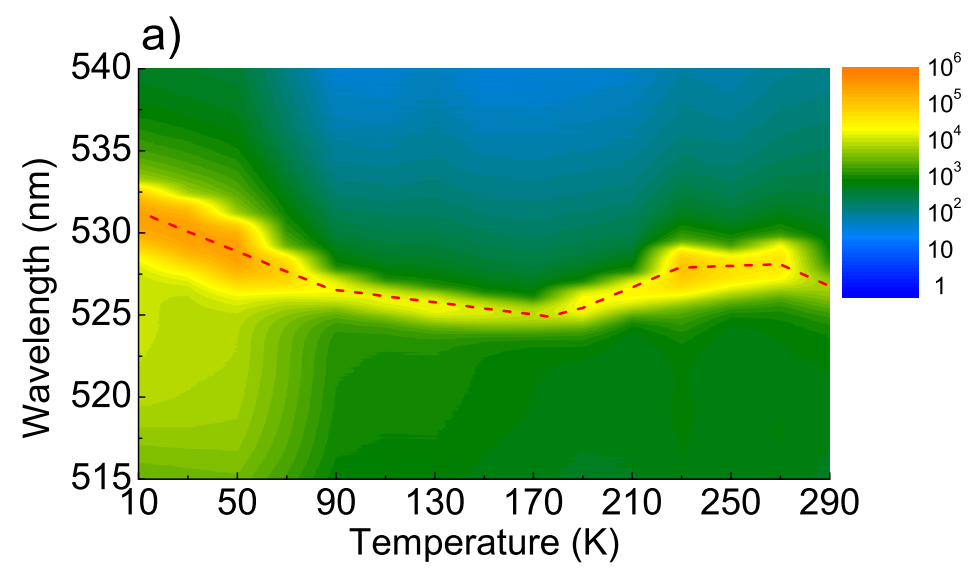

b)

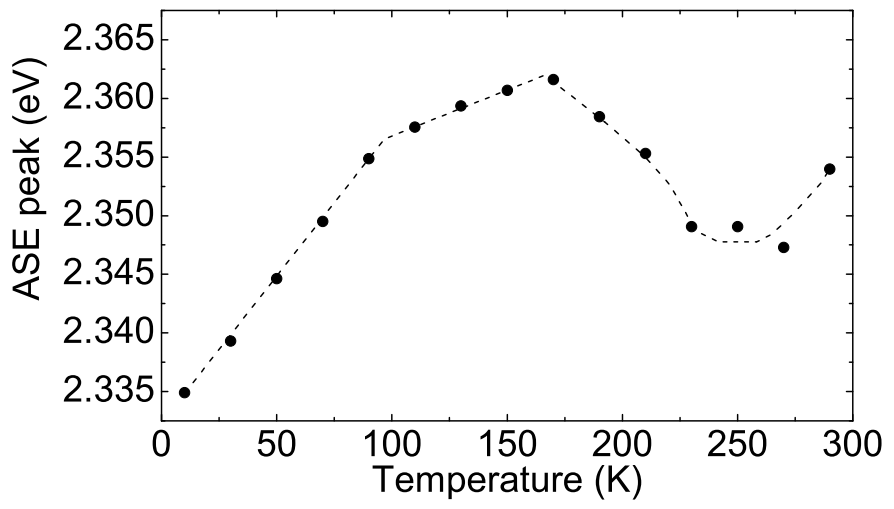

c)

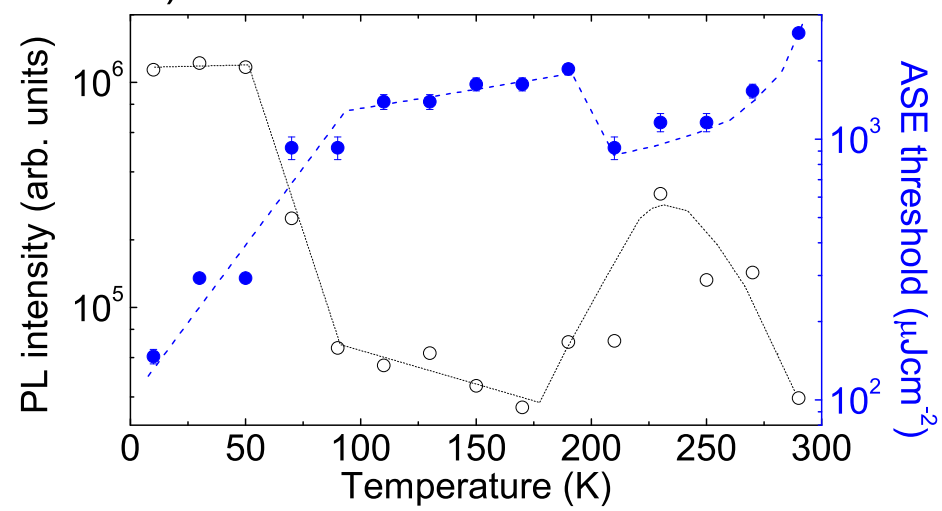

Figure 2: a): 2D map of the PL spectra temperature dependence above the ASE threshold. The red dashed line evidences the peak wavelength position. b): Temperature dependence of the ASE peak energy. c): Temperature dependence of the total intensity and of the ASE threshold.

When the temperature increases clear variations of the lineshape, the PL peak energy, and the PL intensity are observed. In particular we observe that the intensity of the sharp peak dominating the PL at $\mathrm{T}=10 \mathrm{~K}$ strongly decreases at $30 \mathrm{~K}$, giving rise to a much broader 

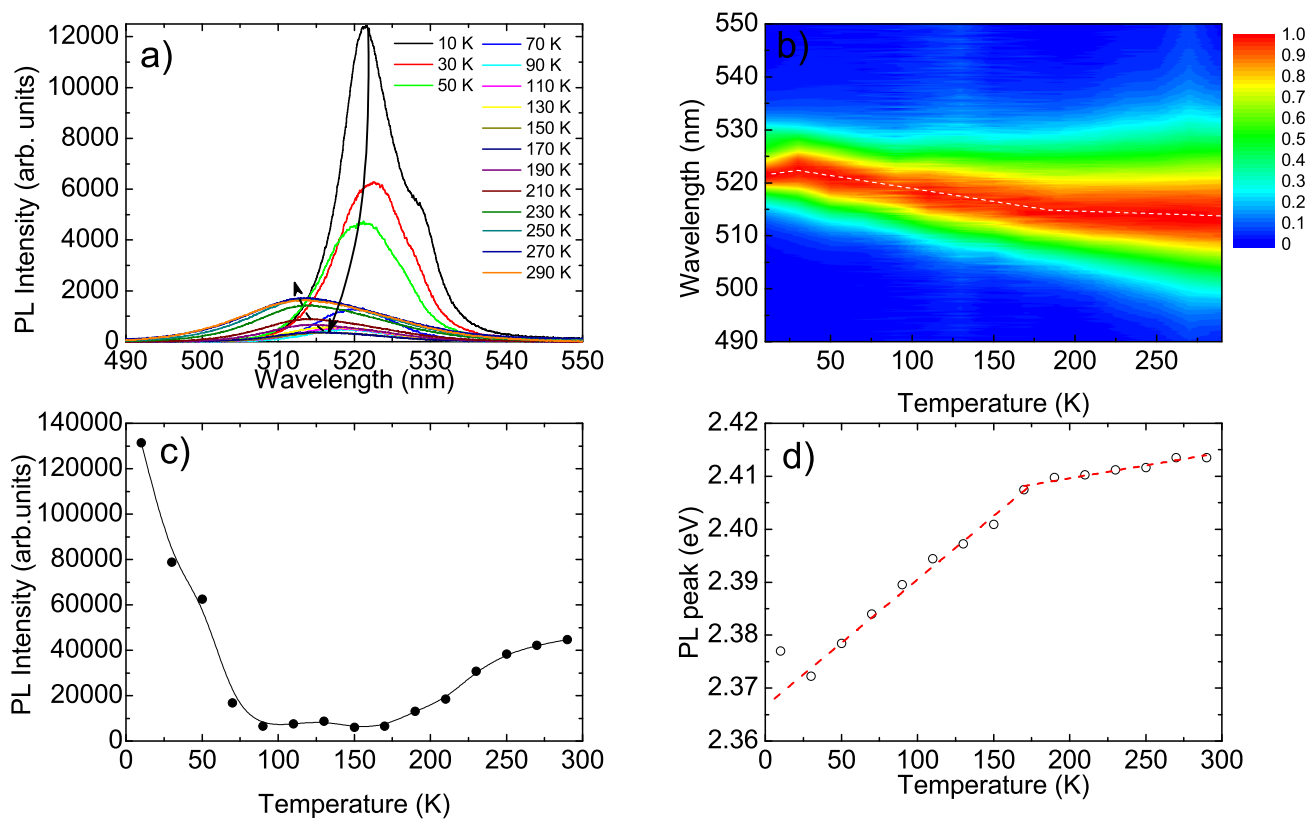

Figure 3: a): Temperature dependence of the PL spectra temperature below the ASE threshold. b): 2 D map of the spectra (normalized to the peak value) evidencing the temperature dependent shift of the peak wavelength (dotted line). c): Temperature dependence of the total intensity. d): Temperature dependence of the PL peak energy. The lines are the best fit curves of a linear fit.

and featureless spectrum, redshifted of $1.1 \mathrm{~nm}(5 \mathrm{meV})$ (see Fig.3 d), and with a FWHM increased to $11.4 \mathrm{~nm}(51.7 \mathrm{meV})$. When the temperature is further increased the following features are observed:

1) The PL peak energy (see Fig.3 b and d) shows a linear blue shift with a best fit slope of $239 \pm 13 \mu \mathrm{eV} / \mathrm{K}$ up to $170 \mathrm{~K}$, with a clear slope reduction down to $48 \pm 5 \mu \mathrm{eV} / \mathrm{K}$ at higher temperatures;

2) the PL intensity (see Fig. 3 c) initially shows a very strong intensity decrease between 10 $\mathrm{K}$ and $90 \mathrm{~K}$ (about $95 \%$ intensity reduction), followed by a much weaker intensity decrease up to $170 \mathrm{~K}$, and then by a very clear recovery ( 7 times) up to $290 \mathrm{~K}$;

The linear blue-shift of the PL peak energy has been already demonstrated in many perovskite materials, ${ }^{14,16,17,20,21,25}$ evidencing a different behavior with respect to typical inorganic semiconductors, whose PL peak energy typically decreases as the temperature in- 
creases. ${ }^{28}$ This behavior evidences that in these materials the blue-shift induced by thermal expansion $^{29}$ dominates over the band gap renormalization due to the increase of the interaction with phonons (leading to a red-shift). ${ }^{30}$ The best fit value of the peak energy blueshift up to $170 \mathrm{~K}$ is in good agreement with the one found in similar NCs in literature, ${ }^{17}$ while the slope variation above $170 \mathrm{~K}$ has not been observed to date.

Concerning the PL intensity we observe that previous investigations on $\mathrm{CsPbBr}_{3} \mathrm{NC}-$ sallowed to observe an intensity quenching as the temperature increases, generally around $10 \%$ in NCs realized at high temperature, both in thin films ${ }^{17}$ and in inert matrix, ${ }^{16}$ and much higher in NCs synthesized at room temperature. ${ }^{31,32}$ A PL intensity increase with the temperature has been instead recently observed in formamidinium lead triiodide NCs, and ascribed to thermally induced exciton detrapping from surface state, while the same material in thin films only shows a strong quenching. ${ }^{14}$

The investigation of the PL spectra temperature dependence, based on a multigaussian fitting (see Supplementary informations), evidences that the spectra can be reproduced with the superposition of two gaussian curves up to $170 \mathrm{~K}$, while a third gaussian at high wavelength is needed to reproduce the spectra at higher temperatures (see Fig. S3). The presence of distinct peaks in the PL spectra of perovskites has been often reported to date, and ascribed to a wide variety of processes, like the coexistence of a majority crystalline phase with inclusions of a different crystalline phase, ${ }^{15,22,23,33}$ emission of free excitons and donor-acceptor pairs, ${ }^{23}$ or emission of free excitons and bound excitons. ${ }^{9,34}$ In particular the coexistence of a narrow PL peak and a broader red shifted peak has been observed at $\mathrm{T}=10 \mathrm{~K}$ in $\mathrm{CsPbBr}_{3} \mathrm{NCs}_{\text {films }}{ }^{35}$ and in microstructures of $\mathrm{FAPbBr}_{3}$, and ascribed to the coexistence of free excitons and bound excitons emission, respectively. ${ }^{34}$ According to this attribution the lineshape variation between $10 \mathrm{~K}$ and $30 \mathrm{~K}$ could be ascribed to a variation of the peak at high energy from a narrow free exciton peak at $521 \mathrm{~nm}$ to a broader bound exciton peak at $524.4 \mathrm{~nm}$, with a increase of the total linewidth and a decrease of the emission intensity. In the temperature range between $30 \mathrm{~K}$ and $90 \mathrm{~K}$ both peaks show 
a strong and quantitatively comparable quenching (see Fig. S4), leading to a total strong PL intensity decrease but a constant relative contribution to the total intensity (see Fig. S5). This behavior suggests that the two gaussian peaks simply come from a non gaussian PL lineshape, showing a similar temperature evolution across the emission spectral range, due to energy migration/transfer from the smaller nanocrystal to the larger ones, within the Gaussian size distribution (see Fig. S1 b), as also observed in $\mathrm{CsPBBr}_{3} \mathrm{NCs}_{\text {solutions. }}{ }^{36}$ As the temperature increases a clear blue shift of the low wavelength peak (peak 1) is observed, while the high wavelength peak (peak 2) shows a weak redshift (see Fig. S6).

The intensity quenching in this temperature range evidences a trapping by non shallow radiative defects, that is always thermally activated. ${ }^{26} \mathrm{~A}$ further explanation could be a temperature induced increase of the self absorption, that produces particularly evident effects in the used waveguide collection geometry, due to the thermal broadening of the exciton absorption peak. ${ }^{19}$ In the range between $90 \mathrm{~K}$ and $170 \mathrm{~K}$ the weak total intensity decrease is due to the interplay between the intensity decrease of peak 1 (3.7 times) and a strong (7.4 times) intensity recovery of peak 2 , leading to a progressive increase of the peak 2 relative contribution to the total intensity. This behavior evidences the formation of a new specie emitting in the low energy side of the spectra. Above $170 \mathrm{~K}$ a further emission contribution in the low energy side of the spectrum appears, described by peak 3, accounting for about $20 \%$ of the total emission intensity, with a comparable relative intensity decrease of peak 1. This new emission feature initially redshifts up to $210 \mathrm{~K}$, and then blueshifts.

This analysis thus evidences that the PL and ASE variation around $90 \mathrm{~K}$ and 170-190 K are related to the formation of two new emitting species in the high wavelength range of the spectra, described by a peak initially red-shifting and then blue shifting.

We also observe that the intensity variations of the gaussian peaks is strictly related to the peak wavelength temperature dependence, both of the spontaneous emission and of the ASE band (see Fig. S6). In particular both PL and ASE peaks show an identical blue shift up to $90 \mathrm{~K}$, determined by the temperature dependence of the peak wavelength of peak 1 . 
Above $90 \mathrm{~K}$, the decrease of the ASE blueshift slope and of the ASE threshold increase takes place in correspondence of the peak 2 intensity recovery, reflecting the strong dependence of the ASE band on the variation of the emission properties in the low energy tail of the PL spectrum, where self absorption is negligible. A remarkable correlation is also observed between the ASE peak wavelength and the peak wavelength of peak 3, above $170 \mathrm{~K}$, clearly evidencing that ASE originates from the emitter at lower energy. The general intensity recovery and improvement of the ASE properties above $170 \mathrm{~K}$ is in apparent contrast with previous reports of PL quenching of the PL intensity of $\mathrm{CsPbBr}_{3} \mathrm{NCs}$, both in neat films ${ }^{17}$ and in inert polymer matrix, ${ }^{16}$ ascribed to exciton thermal dissociation. We ascribe this effect to the interplay between emission quenching due to exciton dissociation and a dominating reduction of self absorption due to the decrease of the exciton absorption below the energy gap, overall leading to an increase of the intensity collected from the waveguide edge. ${ }^{37}$

Concerning the attribution of the temperature induced formation of emitting states at low energy, we exclude any role of macroscopic crystalline phase transitions, as $\mathrm{CsPbBr}_{3}$ is characterized, both in bulk and in NCs, by an average orthorhombic crystal structure, ${ }^{38-40}$ while phase transitions toward tetragonal and cubic phases only take place above room temperature. ${ }^{38}$ We also exclude relevant roles of trapping and detrapping processes, recently observed to affect the ASE properties of $\mathrm{CsPbBr}_{3}$ bulk polycrystalline thin films, ${ }^{26}$ due to the observed complex temperature dependence of the PL lineshape. While in general a thermally activated trapping (detrapping) could lead to a temperature dependent PL quenching (enhancement) the full explanation of our results would require a too complex interplay between trapping and detrapping processes, i. e. a trapping with a very low activation energy leading to a global PL quenching up to $90 \mathrm{~K}$, followed by a simultaneous trapping induced PL quenching at low wavelength and a detrapping at high wavelength between $90 \mathrm{~K}$ and $170 \mathrm{~K}$, and finally a general detrapping induced PL increase above 170 $\mathrm{K}$, in contrast with the PL quenching reported in previous experiments. ${ }^{16,17}$

The thermal activation of the increase of emission in the high wavelength range, with the 
simultaneous intensity decrease at short wavelength is instead qualitatively consistent with the thermal activation of energy migration/transfer from small nanocrystals (emitting at short wavelength) toward the large ones (emitting at high wavelength) within the nanocrystal size distribution (see Fig. S1). In this case at low temperature the small available thermal energy should prevent long range energy migration, only allowing spontaneous energy migration toward low energy (down-hill migration),${ }^{41}$ resulting in a temperature independent lineshape. As the temperature increases the thermal activation of energy migration toward high-energy sites (up-hill migration) allows a long range migration toward deeper energy minima, thus overall increasing the emission at high wavelength at expenses of the one at low wavelength. Our results are anyway not consistent with this expected dependence in many points. First of all the low temperature data (up to $90 \mathrm{~K}$ ) evidence the presence of two peaks with similar intensity decrease up to $90 \mathrm{~K}$, compatible with the absence of thermally activated energy migration. However this does not lead to a temperature independent lineshape, as we observe a continuous broadening at high wavelength evidenced by the peak 2 redshift. A further important difference between our data and the expected behavior of thermally activated energy migration is the presence of two temperatures at which both the spontaneous emission and the ASE temperature dependences strongly change, while long range energy migration typically shows a single activation energy.

Our results are instead consistent with the recent attribution of the complex X-ray spectra of $\mathrm{CsPbBr}_{3} \mathrm{NCs}$ to the presence within the $\mathrm{NC}$ of a nearly ordered orthorombic subdomains, separated by a network of twin boundaries, with a number increasing with the temperature. ${ }^{40}$ Similar conclusions have been also recently obtained in order to explain the presence of multipeak PL spectra in polycrystalline solution processes perovskites thin films, attributing the subdomains to different order level of the organic component. ${ }^{21}$ The presence of two clear transition temperatures between the different observed emission regimes, namely $90 \mathrm{~K}$ and $170 \mathrm{~K}$, suggest that the twin boundaries formation requires specific value of the lattice parameters, that are temperature dependent due to the thermal expansion. Moreover the 
temperature dependence of the peak wavelength of peak 2 up to $110 \mathrm{~K}$, and of peak 3 and of the ASE peak above $170 \mathrm{~K}$, showing a red-shift followed by a blue-shift, is consistent with an initial temperature induced increase of the subdomains dimensions, followed by a dominant contribution of the lattice expansion induced blue-shift. The formation of these disordered domains results in the increase of the emission at low energy, ${ }^{21}$ and clearly modifies the PL spectra, strongly affecting the ASE properties.

\section{Conclusions}

In conclusions we investigated the temperature dependence of the spontaneous and stimulated emission of $\mathrm{CsPbBr}_{3} \mathrm{NCs}$ in thin films, evidencing the presence of three different emission regimes, ascribed to the formation and the temperature evolution of partially disordered sub-domains, strongly affecting the macroscopic ASE properties of the films. Our results improve the understanding of the perovskite NCs light amplification properties, demonstrating that the total ASE properties of NCs thin films are mainly determined by the emission properties of a minority distribution of sub-domains emitting at low energy.

\section{Supporting Informations}

Nanocrystals materials and synthesis details; TEM characterization of the shape and the size of the NCs (Fig. S1); PLQY in solution; SEM and fluorescence microscopy of the film morphology (Fig. S2); details of the multigaussian fitting; temperature dependence of the number of gaussian peaks (Fig. S3); Temperature dependence of the peaks intensity (Fig. S4), relative intensity (Fig. S5) and peak wavelength (Fig. S6). 


\section{Acknowledgments}

M.K. acknowledges financial support from the European Union through the FP7 (ERC Starting Grant NANOSOLID, GA No. 306733).

\section{References}

(1) National Renewable Energy Laboratory. Research Cell Record Efficiency Chart. https://www.nrel.gov/pv/assets/images/efficiency-chart.png (accessed July 4, 2017).

(2) Edri, E.; Kirmayer, S.; Mukhopadhyay, S.; Gartsman, K.; Hodes, G.; Cahen, D. Elucidating the Charge Carrier Separation and Working Mechanism of CH3NH3PbI3-xClx Perovskite Solar Cells. Nat. Commun. 2014, 5, 4461.

(3) Shi, D.; Adinolfi, V.; Comin, R.; Yuan, M.; Alarousu, E.; Buin, A.; Chen, Y.; Hoogland, S.; Rothenberger, A.; Katsiev, K. et al. Low Trap-State Density and Long Carrier Diffusion in Organolead Trihalide Perovskite Single Crystals. Science 2015, 347, 519522.

(4) Protesescu, L.; Yakunin, S.; Bodnarchuk, M. I.; Krieg, F.; Caputo, R.; Hendon, C. H.; Yang, R. X.; Walsh, A.; Kovalenko, M. V. Nanocrystals of Cesium Lead Halide Perovskites (CsPbX3, X = Cl, Br, and I): Novel Optoelectronic Materials Showing Bright Emission with Wide Color Gamut. Nano Lett. 2015, 15, 3692-3696.

(5) Noh, J. H.; Im, S. H.; Heo, J. H.; Mandal, T. N.; Seok, S. I. Chemical Management for Colorful, Efficient, and Stable Inorganic Organic Hybrid Nanostructured Solar Cells. Nano Lett. 2013, 13, 1764-1769.

(6) Protesescu, L.; Yakunin, S.; Kumar, S.; Bär, J.; Bertolotti, F.; Masciocchi, N.; Guagliardi, A.; Grotevent, M.; Shorubalko, I.; Bodnarchuk, M. I. et al. Dismantling 
the "Red Wall" of Colloidal Perovskites: Highly Luminescent Formamidinium and Formamidinium-Cesium Lead Iodide Nanocrystals. ACS Nano 2017, 11, 3119-3134.

(7) Nedelcu, G.; Protesescu, L.; Yakunin, S.; Bodnarchuk, M. I.; Grotevent, M. J.; Kovalenko, M. V. Fast Anion-Exchange in Highly Luminescent Nanocrystals of Cesium Lead Halide Perovskites (CsPbX3, X = Cl, Br, I). Nano Lett. 2015, 15, 5635-5640.

(8) Akkerman, Q. A.; D'Innocenzo, V.; Accornero, S.; Scarpellini, A.; Petrozza, A.; Prato, M.; Manna, L. Tuning the Optical Properties of Cesium Lead Halide Perovskite Nanocrystals by Anion Exchange Reactions. J. Am. Chem. Soc. 2015, 137, 1027610281.

(9) Xing, G.; Nripan, M.; Lim, S. S.; Yantara, N.; Liu, X.; Sabba, D.; Grätzel, M.; Mhaisalkar, S.; Sum, T. C. Low-Temperature Solution-Processed Wavelength-Tunable Perovskites for Lasing. Nat. Mater. 2014, 13, 476-480.

(10) Yakunin, S.; Protesescu, L.; Krieg, F.; Bodnarchuk, I., Maryna; Nedelcu, G.; Humer, M.; De Luca, G.; Fiebig, M.; Heiss, W.; Kovalenko, M. V. Low-Threshold Amplified Spontaneous Emission and Lasing From Colloidal Nanocrystals of Caesium Lead Halide Perovskites. Nat. Commun. 2015, 6, 8056.

(11) Deschler, F.; Price, M.; Pathak, S.; Klintberg, L. E.; Jarausch, D.-D.; Higler, R.; Hüttner, S.; Leijtens, T.; Stranks, S. D.; Snaith, H. J. et al. High Photoluminescence Efficiency and Optically Pumped Lasing in Solution-Processed Mixed Halide Perovskite Semiconductors. J. Phys. Chem. Lett. 2014, 5, 1421-1426.

(12) Dhanker, R.; Brigeman, A. N.; Larsen, A. V.; Stewart, R. J.; Asbury, J. B.; Giebink, N. C. Random Lasing in Organo-Lead Halide Perovskite Microcrystal Networks. Appl. Phys. Lett. 2014, 105, 151112.

(13) Whitworth, G. L.; Harwell, J. R.; Miller, D. N.; Hedley, G. J.; Zhang, W.; Snaith, H. J.; 
Turnbull, G. A.; Samuel, I. D. W. Nanoimprinted Distributed Feedback Lasers of Solution Processed Hybrid Perovskites. Opt. Express 2016, 24, 23677-23684.

(14) Fang, H.-H.; Protesescu, L.; Balazs, D. M.; Adjokatse, S.; Kovalenko, M. V.; Loi, M. A. Exciton Recombination in Formamidinium Lead Triiodide: Nanocrystals versus Thin Films. Small 2017, 13, 1700673.

(15) Wu, K.; Bera, A.; Ma, C.; Du, Y.; Yang, Y.; Li, L.; Wu, T. Temperature-Dependent Excitonic Photoluminescence of Hybrid Organometal Halide Perovskite Films. Phys. Chem. Chem. Phys. 2014, 16, 22476-22481.

(16) Diroll, B. T.; Nedelcu, G.; Kovalenko, M. V.; Schaller, R. D. High-Temperature Photoluminescence of CsPbX3 (X = Cl, Br, I) Nanocrystals. Adv. Funct. Mater. 2017, 27, 1606750.

(17) Li, J.; Yuan, X.; Jing, P.; Li, J.; Wei, M.; Hua, J.; Zhao, J.; Tian, L. TemperatureDependent Photoluminescence of Inorganic Perovskite Nanocrystal Films. RSC Adv. 2016, 6, 78311-78316.

(18) Sestu, N.; Cadelano, M.; Sarritzu, V.; Chen, F.; Marongiu, D.; Piras, R.; Mainas, M.; Quochi, F.; Saba, M.; Mura, A. et al. Absorption F-Sum Rule for the Exciton Binding Energy in Methylammonium Lead Halide Perovskites. J. Phys. Chem. Lett. 2015, 6, $4566-4572$.

(19) D’ Innocenzo, V.; Grancini, G.; Alcocer, M. J. P.; Kandada, A. R. S.; Stranks, S. D.; Lee, M. M.; Lanzani, G.; Snaith, H. J.; Petrozza, A. Excitons Versus Free Charges in Organo-Lead Tri-Halide Perovskites. Nat. Commun. 2014, 5, 3586.

(20) Li, D.; Wang, G.; Cheng, H.-C.; Chen, C.-Y.; Wu, H.; Liu, Y.; Huang, Y.; Duan, X. SizeDependent Phase Transition in Methylammonium Lead Iodide Perovskite Microplate Crystals. Nat. Commun. 2016, \%, 11330. 
(21) Dar, M. I.; Jacopin, G.; Meloni, S.; Mattoni, A.; Arora, N.; Boziki, A.; Zakeeruddin, S. M.; Rothlisberger, U.; Grätzel, M. Origin of Unusual Bandgap Shift and Dual Emission in Organic-Inorganic Lead Halide Perovskites. Sci. Adv. 2016, 2, e1601156.

(22) Wehrenfennig, C.; Liu, M.; Snaith, H. J.; Johnston, M. B.; Herz, L. M. Charge Carrier Recombination Channels in the Low-Temperature Phase of Organic-Inorganic Lead Halide Perovskite Thin Films. APL Mater. 2014, 2, 081513.

(23) Kong, W.; Ye, Z.; Qi, Z.; Zhang, B.; Wang, M.; Rahimi-Iman, A.; Wu, H. Characterization of an Abnormal Photoluminescence Behavior Upon Crystal-Phase Transition of Perovskite CH3NH3PbI3. Phys. Chem. Chem. Phys. 2015, 17, 16405-16411.

(24) Kao, T. S.; Chou, Y.-H.; Chou, C.-H.; Chen, F.-C.; Lu, T.-C. Lasing Behaviors upon Phase Transition in Solution-Processed Perovskite Thin Films. Appl. Phys. Lett. 2014, $105,231108$.

(25) Qin, L.; Lv, L.; Li, C.; Zhu, L.; Cui, Q.; Hu, Y.; Lou, Z.; Teng, F.; Hou, Y. Temperature Dependent Amplified Spontaneous Emission of Vacuum Annealed Perovskite Films. RSC Adv. 2017, 7, 15911-15916.

(26) De Giorgi, M. L.; Perulli, A.; Yantara, N.; Boix, P. P.; Anni, M. Amplified Spontaneous Emission Properties of Solution Processed CsPbBr3 Perovskite Thin Films. J. Phys. Chem. C 2017, 121, 14772-14778.

(27) Brouwer, A. M. Standards for Photoluminescence Quantum Yield Measurements in Solution (IUPAC Technical Report). Pure Appl. Chem. 2011, 83, 2213-2228.

(28) Becker, M.; Fan, H. Y. Optical Properties of Semiconductors. III. Infra-Red Transmission of Silicon. Phys. Rev. 1949, 76, 1531-1532.

(29) Bardeen, J.; Shockley, W. Deformation Potentials and Mobilities in Non-Polar Crystals. Phys. Rev. 1950, 80, 72-80. 
(30) Fan, H. Y. Temperature Dependence of the Energy Gap in Monatomic Semiconductors. Phys. Rev. 1950, 78, 808-809.

(31) Li, X.; Wu, Y.; Zhang, S.; Cai, B.; Gu, Y.; Song, J.; Zeng, H. CsPbX3 Quantum Dots for Lighting and Displays: Room-Temperature Synthesis, Photoluminescence Superiorities, Underlying Origins and White Light-Emitting Diodes. Adv. Funct. Mater. 2016, 26, $2435-2445$.

(32) Wei, S.; Yang, Y.; Kang, X.; Wang, L.; Huang, L.; Pan, D. Room-Temperature and Gram-Scale Synthesis of CsPbX3 (X = Cl, Br, I) Perovskite Nanocrystals with 50-85\% Photoluminescence Quantum Yields. Chem. Commun. 2016, 52, 7265-7268.

(33) Diab, H.; Trippé-Allard, G.; Lédée, F.; Jemli, K.; Vilar, C.; Bouchez, G.; Jacques, V. L.; Tejeda, A.; Even, J.; Lauret, J.-S. et al. Narrow Linewidth Excitonic Emission in OrganicInorganic Lead Iodide Perovskite Single Crystals. J. Phys. Chem. Lett. 2016, 7, 5093-5100.

(34) Dai, J.; Zheng, H.; Zhu, C.; Lu, J.; Xu, C. Comparative Investigation on TemperatureDependent Photoluminescence of CH3NH3PbBr3 and $\mathrm{CH}(\mathrm{NH} 2) 2 \mathrm{PbBr} 3$ Microstructures. J. Mater. Chem. C 2016, 4, 4408-4413.

(35) Lee, S. M.; Moon, C. J.; Lim, H.; Lee, Y.; Choi, M. Y.; Bang, J. Temperature-Dependent Photoluminescence of Cesium Lead Halide Perovskite Quantum Dots: Splitting of the Photoluminescence Peaks of $\mathrm{CsPbBr} 3$ and $\mathrm{CsPb}(\mathrm{Br} / \mathrm{I}) 3$ Quantum Dots at Low Temperature. J. Phys. Chem. C 2017, 121, 26054-26062.

(36) Weerd, C.; Navascues, L.; Zhang, H.; Buma, W.; Nedelcu, G.; Kovalenko, M.; Gregorkiewicz, T. Energy Transfer Between Inorganic Perovskite Nanocrystals. J. Phys. Chem. C 2016, 120, 13310-13315.

(37) Anni, M. Photodegradation Effects on the Emission Properties of an Amplifying 
Poly(9,9-dioctylfluorene) Active Waveguide Operating in Air. J. Phys. Chem. B 2012, $116,4655-4660$.

(38) Rodová, M.; Brozek, J.; Knízek, K.; Nitsch, K. Phase Transitions in Ternary Caesium Lead Bromide. J. Therm. Anal. Calorim. 2003, 71, 667-673.

(39) Cottingham, P.; Brutchey, R. L. On the Crystal Structure of Colloidally Prepared CsPbBr3 Quantum Dots. Chem. Commun. 2016, 52, 5246-5249.

(40) Bertolotti, F.; Protesescu, L.; Kovalenko, M. V.; Yakunin, S.; Cervellino, A.; Billinge, S. J. L.; Terban, M. W.; Pedersen, J. S.; Masciocchi, N.; Guagliardi, A. Coherent Nanotwins and Dynamic Disorder in Cesium Lead Halide Perovskite Nanocrystals. ACS Nano 2017, 11, 3819-3831.

(41) Meskers, S. C. J.; Hübner, J.; Oestreich, M.; Bässler, H. Dispersive Relaxation Dynamics of Photoexcitations in a Polyfluorene Film Involving Energy Transfer: Experiment and Monte Carlo Simulations. J. Phys. Chem. B 2001, 105, 9139-9149. 


\section{TOC Graphic}

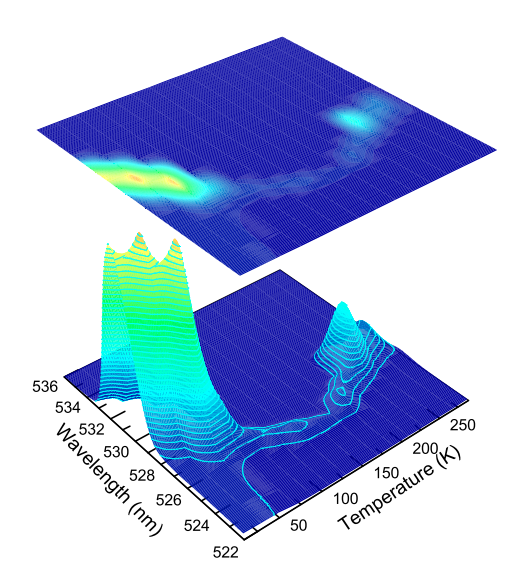

